\title{
A new form of human spatial attention: Automated symbolic orienting
}

\section{Jelena Ristic \& Alan Kingstone}

To cite this article: Jelena Ristic \& Alan Kingstone (2012) A new form of human spatial attention: Automated symbolic orienting, Visual Cognition, 20:3, 244-264, DOI: 10.1080/13506285.2012.658101

To link to this article: http://dx.doi.org/10.1080/13506285.2012.658101

曲 Published online: 22 Feb 2012.

Submit your article to this journal $\sqsubset x$

Џلll Article views: 412

Q View related articles $\square$

Citing articles: 17 View citing articles 4 


\title{
A new form of human spatial attention: Automated symbolic orienting
}

\author{
Jelena Ristic ${ }^{1}$ and Alan Kingstone ${ }^{2}$ \\ ${ }^{1}$ Department of Psychology, McGill University, Montreal, Canada \\ ${ }^{2}$ Department of Psychology, University of British Columbia, \\ Vancouver, Canada
}

\begin{abstract}
The control of human attention is typically conceptualized either in terms of exogenous automatic processes that are driven by external sensory stimulation or endogenous strategic processes that are driven by internal expectancies about events in the environment. However, this classic dichotomy has struggled to explain a wealth of new data demonstrating that behaviourally and biologically relevant visual stimuli, like arrow and eye direction, elicit shifts of spatial attention that on the one hand, appear exogenous, and on the other hand, endogenous. To address this issue, we used a double-cueing task that combined arrows with classic cues known to invoke either exogenous or endogenous orienting. Our data suggest that behaviourally relevant directional cues, like arrows, engage a new form of cortically mediated orienting - automated symbolic orienting - that operates independent of, and in parallel with, the two classic forms of exogenous and endogenous spatial attention.
\end{abstract}

Keywords: Behaviourally relevant cues; Human attention; Spatial orienting.

Theories of human attention are often framed in terms of processes that occur exogenously, driven by external stimulation, or endogenously, driven by internal expectations (Posner, 1978). This conceptual framework has its roots in James' writings on attention (1890) and has underscored a broad range of attention research within recent decades, including behavioural (e.g., Egeth \& Yantis, 1997), neuroimaging (e.g., Corbetta \& Shulman, 2002),

Please address all correspondence to Jelena Ristic, Department of Psychology, McGill University, 1205 Dr. Penfield Ave,, Montreal, QC H3A 1B1, Canada. E-mail: jelena.ristic@mcgill.ca

Supported by NSERC (JR, AK), G. W. Stairs (JR), MSFHR (JR), and SSHRC (AK) funds.

(C) 2012 Psychology Press, an imprint of the Taylor \& Francis Group, an Informa business http://www.psypress.com/viscog

http://dx.doi.org/10.1080/13506285.2012.658101 
developmental (e.g., Brodeur, Trick, \& Enns, 1997), neuropsychological (e.g., Bartolomeo \& Chokron, 2002), and animal studies (e.g., Dorris, Klein, Everling, \& Munoz, 2002). This commonly accepted taxonomy, however, has struggled to account for recent data derived from the model cueing task that has traditionally been used to invoke and measure exogenous and endogenous orienting. Specifically, when behaviourally and biologically relevant symbolic stimuli, such as human eyes or pointing arrows, are used within the cueing task, the data cannot be fully explained as either exogenous or endogenous attentional selection (e.g., Bonato, Priftis, Marenzi, \& Zorzi, 2008; Gibson \& Kingstone, 2006). The aim of the present study is to examine and attempt to resolve this fundamental discrepancy between theory and data in the field of attention.

The classic attention cueing paradigm applies experimental procedures that are intended to reveal the properties of exogenous and endogenous orienting. Exogenous orienting of spatial attention occurs when attention is drawn to a spatial location by a simple peripheral stimulus, such as an abrupt luminance change (Posner, 1980). To minimize the role of cognitive factors, the stimulus cue does not reliably predict where a response target will appear. The sensory cue quickly produces facilitation in response time (RT) for a target that appears at the cued/attended spatial location. This response facilitation is subsequently replaced by a delay in RT for a target at the cued location, revealing an effect coined 'inhibition of return' (IOR; Posner \& Cohen, 1984). Endogenous attention, on the other hand, occurs when attention is directed to a spatial location by a symbolic central stimulus (Jonides, 1981). To enhance the role of strategic factors, the central cue reliably predicts where a response target will appear. In comparison to exogenous orienting, endogenous orienting slowly gives rise to a long lasting RT facilitation at the cued location and does not produce an IOR effect (Taylor \& Klein, 1998).

About a decade ago, several studies reported that when central spatially nonpredictive eye direction (Driver et al., 1999; Friesen \& Kingstone, 1998; Langton \& Bruce, 1999) or arrow direction (Ristic, Friesen, \& Kingstone, 2002; Tipples, 2002) are used within the cueing task, there is rapid RT facilitation, as is typically observed for exogenous orienting, and prolonged RT facilitation at the cued location with no IOR (McKee, Christie, \& Klein, 2007), as is typically observed for endogenous orienting. Since these data were first reported, the field has sought to classify these attention effects as being either under exogenous or endogenous control. Some investigators focused on the similarities that the attentional effects of central nonpredictive cues share with the attentional effects of peripheral nonpredictive cues - in that they both produce rapid facilitation at the cued location - and attributed the observed effects to exogenous orienting (Driver et al., 1999; Friesen \& Kingstone, 1998; but see Green \& Woldorff, 
2012). ${ }^{1}$ Other investigators focused on the similarities that the attentional effects of central nonpredictive cues share with the attentional effects of central predictive cues - in that both effects are elicited by a foveal cue and no IOR is observed - and attributed the observed effects to endogenous orienting (Green \& Woldorff, 2012; Tipples, 2008; Vecera \& Rizzo, 2006). A third group of researchers focused on the similarities that the attentional effects of central nonpredictive cues share with both the effects of peripheral nonpredictive cues and central predictive cues, and attributed the observed effects to a hybrid form of exogenous and endogenous orienting (Klein \& Shore, 2000).

Despite this uncertainty regarding the nature of the orienting effect, investigators have expanded the use of central nonpredictive cues to study attention across different populations and cognitive phenomena (Frischen, Bayliss, \& Tipper, 2007). For instance, in addition to a wealth of studies conducted with adults, the attention effects of nonpredictive eyes and arrows have been measured in infants (e.g., Hood, Willen, \& Driver, 1998), preschool children (e.g., Ristic et al., 2002), ageing adults (e.g., Olk, Hildebrandt, \& Kingstone, 2010), brain-injured patient populations (e.g., Bonato et al., 2008), individuals with autism (e.g., Ristic et al., 2005), and in nonhuman primates (e.g., Deaner \& Platt, 2003). Similarly, these cues have been manipulated in neuroimaging studies of social cognition using both fMRI (e.g., Greene, Mooshagian, Kaplan, Zaidel, \& Iacoboni, 2009; Tipper, Handy, Giesbrecht, \& Kingstone, 2008) and EEG (e.g., Schuller \& Rossion, 2001), and have been used to investigate topics ranging from human emotion (e.g., Graham, Friesen, Fichtenholtz, \& LaBar, 2010) to numerical processing (e.g., Fischer, Castel, Dodd, \& Pratt, 2003). Given the broad appeal and application of central nonpredictive cues to measure the effects of attention, it would be of immense benefit if researchers could gain a clearer sense of what attentional processes are being engaged by these cues. Such knowledge would not only help establish a solid theoretical framework within which to

\footnotetext{
${ }^{1}$ Green and Woldorff (2012) recently argued that the early RT effects observed with central arrow cues cannot reflect reflexive orienting because (1) a RT effect is observed even when cue and target are simultaneous, suggesting impossibly fast reflexive orienting, and (2) flashing the cue at fixation delays the early RT effect, which should be unaffected by cue transients. Both conclusions should be taken with caution. The first conclusion disregards the fact that attentional orienting can occur from the time that a cue appears until a response is made. In Green and Woldorff's study, overall response time exceeded $400 \mathrm{~ms}$, meaning that participants had that long to shift attention from the arrow cue to the target even when cue and target were simultaneous. The second conclusion disregards the well established fact that flashing a stimulus at fixation will itself capture and delay attentional orienting to a peripheral location (e.g., Klein \& Shore, 2000).
} 
understand the extant findings, but it would also aid in formulating critical questions for future investigations.

We tackled this issue by assessing participants' performance when nonpredictive cues are combined with classic cues that are known to engage either exogenous or endogenous orienting. Applying additive factors logic, we reasoned that if two attentional cues influence performance at the same time in an additive fashion, then the underlying attentional mechanisms are operating independently. Conversely, if the cues influence performance in an interactive fashion, then the cues draw on the same underlying mechanisms. This logic yields the following predictions: (1) If the attention effect elicited by a central nonpredictive cue is exogenous, then it should interact with exogenous orienting elicited by a spatially nonpredictive peripheral onset cue and occur in parallel (i.e., not interact) with endogenous orienting elicited by a spatially predictive central cue. (2) If the attentional effect elicited by a nonpredictive central cue is endogenous, then it should occur in parallel with exogenous orienting elicited a spatially nonpredictive peripheral cue and interact with endogenous orienting engaged by a spatially predictive central cue. (3) If the nonpredictive central cue reflects a hybrid of exogenous and endogenous orienting, then it should interact with the attention effects of both cues. There is one other logical possibility, heretofore not considered by the field: (4) A nonpredictive directional central cue produces attentional orienting that is distinct from exogenous and endogenous attention. This fourth possibility predicts that a nonpredictive arrow cue will produce attention effects that do not interact with either the exogenous or endogenous attention effects produced by the classic cues.

To test these hypotheses, we measured observers' performance in two conditions utilizing a double-cueing task in which participants were presented with a central nonpredictive arrow cue combined with either a nonpredictive peripheral cue (exogenous orienting) or a central predictive cue (endogenous orienting). Because a central nonpredictive arrow cue is common to both conditions, the first condition will be referred to as the Nonpredictive Peripheral (NP) cue condition, and the second condition as the Predictive Central (PC) cue condition.

To anticipate the results, we observed significant attention effects in both the NP and PC conditions, but the exogenous and endogenous attention effects did not interact with the attention effects produced by a nonpredictive arrow cue. As we will show, the data provide compelling support for the fourth alternative that central spatially nonpredictive cues produce a form of orienting that is distinct from the classic forms of exogenous and endogenous spatial orienting. 


\section{METHODS}

\section{Participants}

Fifty undergraduates with normal or corrected-to-normal vision.

\section{Apparatus and stimuli}

Experiments were run on a Macintosh computer connected to a 15 -inch monitor. All stimuli were black line drawings presented on white background. Peripheral cues were created by thickening the outline of one of four $2^{\circ} \times 2^{\circ}$ placeholder boxes, each positioned $7.5^{\circ}$ away from central fixation along horizontal and vertical planes, as measured from the centre of the placeholder to the centre of the screen. An arrow cue was created by combining a straight line $\left(2^{\circ}\right)$ with an arrowhead and an arrowtail. The number cues $(9,6,3$, or 1$)$ measured $2^{\circ}$ in height and $1.2^{\circ}(9,6,3)$ or $0.3^{\circ}(1)$ in width. The target was an asterisk, subtending $0.8^{\circ}$ of visual angle, and appeared in the middle of a placeholder. Stimuli, sample sequences of events, and cue validity outcomes for the NP and PC conditions are illustrated in Figures $1 \mathrm{~A}$ and $2 \mathrm{~A}$, respectively.

\section{Design}

Eighteen participants were assigned to the NP group and 32 participants to the PC group to ensure adequate power when spatially predictive cues were used.

Typically, cueing studies only present one cue on any given trial. The few studies using more than one cue have presented the cues in a sequential manner (e.g., Berger, Henik, \& Rafal, 2005; Muller \& Rabbitt, 1989). The advantage of the sequential design is that the workload placed on a participant at any given moment is reduced to that of the standard one-cue study. The limitation is that sequential cues represent a weak test of process independence because participants can perform each form of orienting serially but give the illusion that the two processes are being carried out simultaneously. To control for this possibility we presented the two cues at the same time.

In the NP condition, a spatially nonpredictive peripheral onset and a spatially nonpredictive central arrow were shown. The position of the peripheral cue and the direction of the arrow cue were determined randomly, with the target appearing with equal probability at each possible target location $(p=.25$; Figure 1A). In the PC condition, a spatially predictive central digit and a spatially nonpredictive central arrow were shown. The central number cue indicated the correct target location with .77 probability, 
A. Stimulus presentation sequence
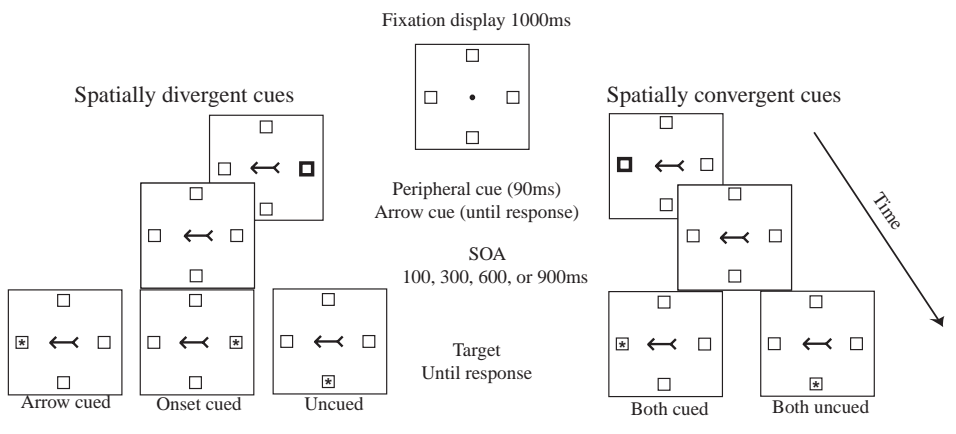

B. RT results

C. Divergent sum vs. Convergent effects
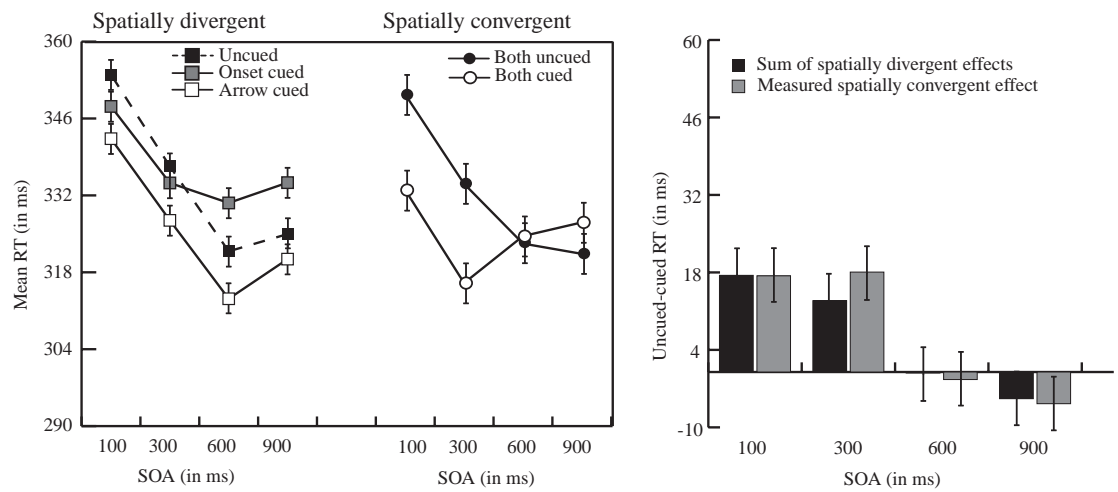

Figure 1. NP (nonpredictive peripheral) condition. (A) Example stimulus presentation sequence and illustration of conditions. A fixation display in duration of $1000 \mathrm{~ms}$ was followed by the presentation of two spatially nonpredictive attentional cues, a peripheral cue created by thickening the outline of one of four possible locations (presented for $90 \mathrm{~ms}$ ), and a central arrow cue created by attaching an arrowhead and an arrowtail to a straight line (presented until response). On any given trial, the two cues could indicate different spatial locations (spatially divergent case) or the same spatial location (spatially convergent case). A single target, demanding a detection response, appeared in one of the four possible locations and remained on the screen until response. Note that the stimuli are not drawn to scale. (B) RT results. Mean correct RT as a function of cues' spatial position (convergent vs. divergent), cue validity (onset cued; arrow cued; uncued; both cued; both uncued), and stimulustarget onset asynchrony (SOA). (C) Divergent sum vs. convergent effects. The magnitude of orienting effect (uncued-cued RT) plotted as a function of SOA when the two cues indicated the same spatial location and the sum of orienting effects when the two cues indicated different spatial locations. Error bars represent the standard error of the difference between means.

while the corresponding arrow cue indicated the correct target location equally often ( $p=.25$; Figure 2A). Spatial predictiveness of the digit cues were set as follows: Number 1 predicted a target occurring at the top, 3 on the right, 6 on the bottom, and 9 on the left. These cue number-target 
A. Stimulus presentation sequence

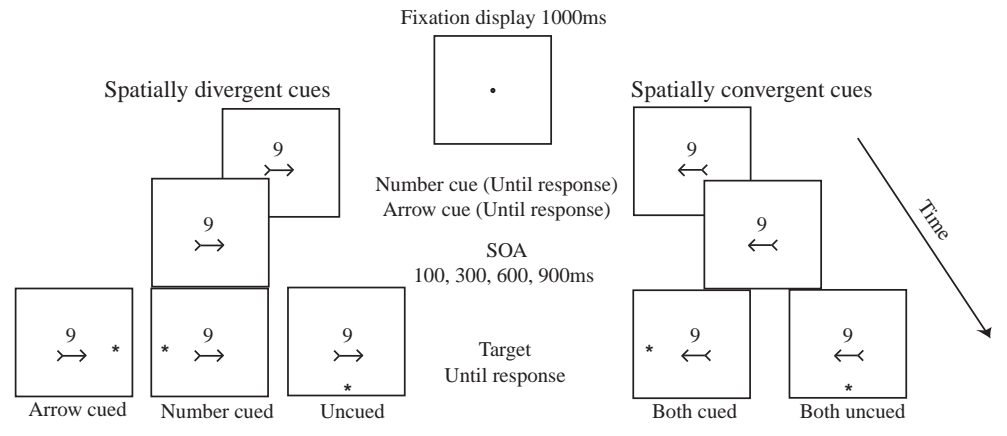

B. RT results

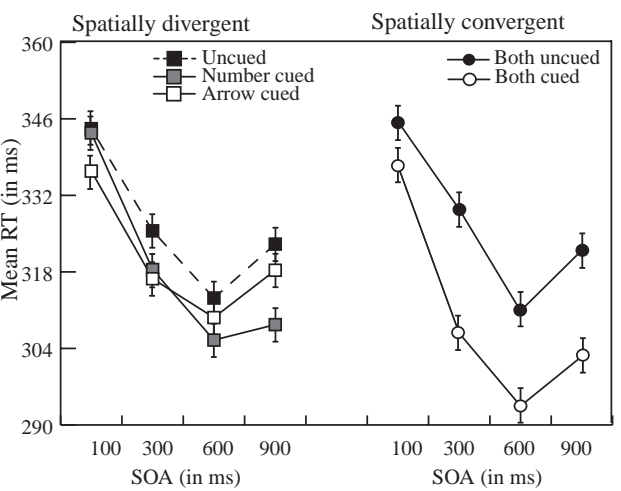

C. Divergent sum vs. Convergent effects

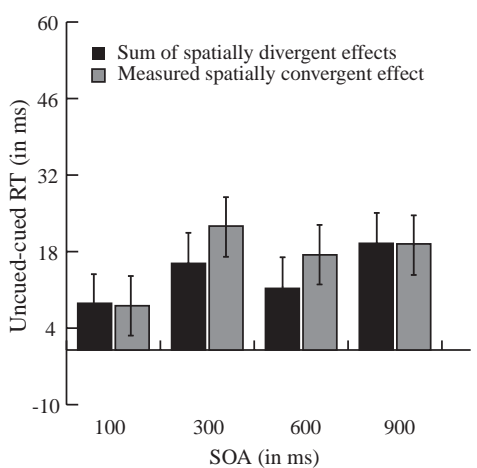

Figure 2. PC (predictive central condition). (A) Example stimulus presentation sequence and illustration of conditions. A fixation display in duration of $1000 \mathrm{~ms}$ was followed by the presentation of two central cues, a central nonpredictive arrow, which indicated one of possible four target locations equally often $(p=25)$, and a central spatially predictive digit $(p=.77)$ whereby the number 1 predicted a target occurring on the top, 3 a target occurring on the right, 6 a target occurring on the bottom, and 9 a target occurring on the left of fixation in $77 \%$ of trials. On any given trial, the two cues could indicate different spatial locations (spatially divergent case) or the same spatial location (spatially convergent case). A single target, demanding a detection response, appeared in one of the four possible locations and remained on the screen until response. Note that the stimuli are not drawn to scale. (B) RT results. Mean correct RT as a function of cues' spatial position (convergent vs. divergent), cue validity (onset cued; arrow cued; uncued; both cued; both uncued), and stimulus-target onset asynchrony (SOA). (C) Divergent sum vs. convergent effects. The magnitude of orienting effect (uncued-cued RT) plotted as a function of SOA when the two cues indicated the same spatial location and the sum of orienting effects when the two cues indicated different spatial locations. Error bars represent the standard error of the difference between means.

relations have been shown to require endogenous attention in order for spatial orienting to occur (Ristic \& Kingstone, 2006). In the PC condition the digit was positioned above fixation and the arrow was positioned below 
fixation at an equal distance of $3.5^{\circ}$ of visual angle from the centre of the number cue to the centre of an arrow cue.

\section{Procedure}

Each trial began with a presentation of a $1000 \mathrm{~ms}$ fixation display. Then, the two cues appeared indicating either two different spatial locations (spatially divergent cues) or the same spatial location (spatially convergent cues). Preserving the typical stimulus presentation sequences, peripheral cues were presented for $90 \mathrm{~ms}$ (Posner, 1980), while central cues (arrow and digit) remained on the screen for the duration of the trial (Ristic, Wright, \& Kingstone, 2006). Following a randomly selected SOA of 100, 300, 600, or $900 \mathrm{~ms}$, a target demanding a detection response appeared at one of four possible target locations (left, right, up or down). The trial was terminated on response or after $2600 \mathrm{~ms}$ had elapsed, whichever came first. RT was based on keyboard responses and was measured from target onset. The intertrial interval was $675 \mathrm{~ms}$. Randomly, on approximately $6 \%$ of the trials, a target was not presented and participants were required to withhold a keypress response.

Participants were seated and centred with respect to the computer screen at an approximate distance of $57 \mathrm{~cm}$. Participants were instructed to maintain central fixation and to press the spacebar as fast and as accurately as possible when they detected the onset of the target. All participants were informed about, and it was confirmed that they understood, the spatial predictiveness of each cue.

Cue direction, target position, and SOA were presented equally and in random order. Each participant completed 10 practice trials and a total of 960 trials divided into 16 blocks.

\section{RESULTS}

Anticipations, defined as RTs less than $100 \mathrm{~ms}(\mathrm{NP}=0.63 \% ; \mathrm{PC}=0.91 \%)$, timed out responses, defined as RTs above $1000 \mathrm{~ms}(\mathrm{NP}=0.15 \%$; PC $0.19 \%)$ and incorrect key presses $(\mathrm{NP}=0.01 \%$ and $\mathrm{PC}=0.01 \%)$ on target present trials were infrequent. Similarly, false alarms on target absent trials were rare $(\mathrm{NP}=0.92 \% ; \mathrm{PC}=1.79 \%)$. All incorrect trials were removed from subsequent analyses.

If the two cues produce independent attention effects, then (1) no interference between the cues should be observed when they are divergent, and (2) the sum of the orienting effects for each of the cues observed in the spatially divergent situation should closely approximate the magnitude of the attention effect observed in the spatially convergent situation. 


\section{NP (nonpredictive peripheral) condition}

Mean RTs for spatially divergent and spatially convergent conditions are illustrated in Figure 1B.

Divergent cues. Two separate within-subject ANOVAs with cue validity and SOA compared the mean RTs for peripheral box and central arrow cues in the spatially divergent condition. For peripheral cues, a significant main effect of SOA, $F(3,51)=21.4, p<.0001$, reflecting a classic foreperiod effect (Bertelson, 1967) and an interaction between cue validity and SOA, $F(3,51)=3.6, p<.05$, reflecting the emergence of an IOR effect were reliable. For arrow cues, significant main effects of SOA, $F(3,51)=27.6, p<.0001$, and cue validity, $F(1,17)=16.6, p<.001$, with no interactions $(p>.3)$ were observed.

Convergent cues. A similar ANOVA was conducted on the spatially convergent condition. This analysis revealed a significant main effect of SOA, $F(3,51)=6.2, p<.05$, and a cue validity $\times$ SOA interaction, $F(3,51)=6.1, p<.05$, indicating that the early RT facilitation effect is later abolished, consistent with the emergence of an IOR effect at the cued location that is triggered by the nonpredictive peripheral cue.

Divergent sum vs convergent cue effects. To determine whether the sum of the orienting effects for the spatially divergent cues closely approximates the magnitude of the orienting effect for the spatially convergent cues, for each SOA we computed the sum of the orienting effects (uncued-cued RT) for the two divergent cues and compared that against the orienting effect elicited by convergent cues. As suggested by Figure $1 \mathrm{C}$, a $4(\mathrm{SOA}) \times 2$ (divergent sum vs. convergent) ANOVA revealed that for both divergent and convergent effects the magnitude of the cueing effect declined as a function of SOA, $F(3,51)=7.9, p<.001$, reflecting the emergence of an IOR at the longer SOAs. Most critically, the sum of the divergent cueing effects and the convergent cueing effect mirrored one another across each of the SOA intervals, resulting in no main effect or interaction involving the divergent and convergent effects (both $F \mathrm{~s}<1$ ). In short, the effects of the two cues were additive.

\section{PC (predictive central) condition}

Mean RTs for spatially divergent and spatially convergent conditions are illustrated in Figure 2B. 
Divergent cues. Two separate within-subject ANOVAs with cue validity and SOA compared the mean RTs for predictive number and nonpredictive central arrow cues in the spatially divergent condition. For nonpredictive arrows, significant main effects of SOA, $F(3,90)=17.7, p<.0001$, and cue validity, $F(3,90)=4.3, p<.05$, with no interactions $(F<1)$ were observed, indicating that the cueing effects were reliable and steady across all SOAs. For predictive numbers, the ANOVA revealed significant main effects of SOA, $F(3,90)=35.4, p<.0001$, and cue validity, $F(1,30)=11.6, p<.01$, as well as a reliable interaction between SOA and cue validity, $F(3,90)=4.2$, $p<.01$, consistent with the observation that the cueing effect was absent at the shortest SOA and present thereafter.

Convergent cues. An ANOVA conducted on the spatially convergent condition revealed significant effects of SOA, $F(3,90)=33.4, p<.0001$, and cue validity, $F(1,30)=32, p<.0001$, with no interactions, $F(3,90)=2, p>.1$, indicating that when the two cues indicated the same location, the cueing effect was significant and steady across all SOAs, reflecting an additive combination of endogenous orienting elicited by spatially predictive number cues and nonpredictive central arrow cues.

Divergent sum vs convergent cue effects. As before, we compared the magnitude of the sum of the cueing effects for divergent cues against the magnitude of the cueing effects for convergent cues. As illustrated in Figure $2 \mathrm{C}$, the sum of the divergent cueing effects closely mirrored the rise and fall of the convergent cueing effects across SOAs. Consistent with these observations, the within-subject ANOVA indicated that there were no reliable main effects $(F<1)$ or interactions $(F<1)$ involving summed and convergent effects. Thus, we find that at each of the four SOAs, a nonpredictive arrow cue produces a significant and reliable attention effect that is independent of, and additive with, the endogenous cueing effect produced by a predictive number cue.

\section{Between cue contrasts}

We further tested the finding of independence between the orienting effects by comparing the attention effect of an arrow cue in the NP condition against the attention effect of an arrow cue in the PC condition. If the effects of the arrow cue are truly independent of the other cues, the cueing effect of a nonpredictive arrow should be the same in the NP and PC conditions.

Figure 3 shows that the attention effects of arrow cues are nearly identical in the NP and PC conditions, both in terms of absolute RTs and with regard to the overall pattern of performance. Whether paired with exogenous or endogenous cues, a nonpredictive arrow cue produces a pattern of rapid 


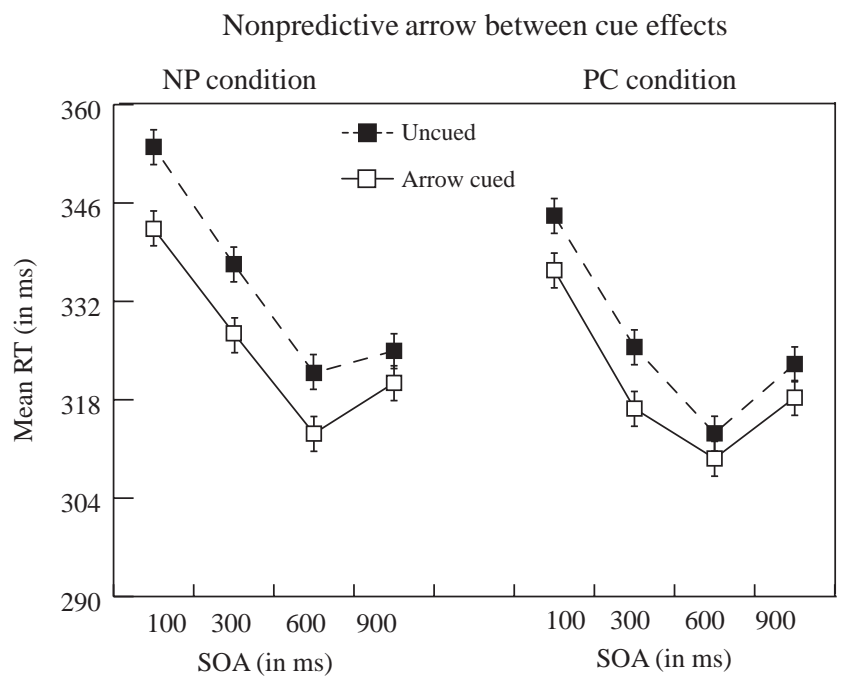

Figure 3. Between cue effects. Mean correct RT as a function of cue validity and SOA for the nonpredictive arrow condition across the NP and PC cue conditions. Error bars represent the standard error of the difference between means.

facilitation that persists across all SOA durations. A mixed ANOVA with cue condition (NP vs. PC) as a between-subject factor, and cue validity and SOA as within-subject factors, confirmed that this pattern is the same for both the NP and PC pairings. The only significant effects to emerge were those that one would expect: cue validity, $F(1,47)=12, p<.01$, and SOA, $F(3,141)=35.3, p<.0001$; all other $F \mathrm{~s}<1$. These data provide a compelling final line of evidence that the attention effect of a nonpredictive arrow cue is independent of the exogenous and endogenous attention effects engaged by classic nonpredictive peripheral cues and predictive central cues, respectively.

\section{Control analyses}

Although our results strongly support the conclusion that orienting elicited by central spatially nonpredictive cues is independent from orienting elicited by classic cues, one might question if our results could arise, at least in part, (1) as an artefact of stimulus repetition or (2) because participants choose to orient attention in response to one cue on some trials and the other cue on the other trials thereby creating the false impression, when the data are combined, that the effects are carried out concurrently without interference. Both of these alternative explanations are not supported by subsequent analyses of the data. First, we examined whether the RT effects were influenced by the repetition of the target at each target location (e.g., Posner $\&$ Cohen, 1984). We found that this variable did not influence the outcome 
of the experiments. When trials in which the target occurred at the same location in two or more consecutive trials (i.e., repeated trials: $29.63 \%$ of trials in the NP condition and $30.62 \%$ of trials in the PS condition) were removed from the analyses, the same data pattern was observed as when they were included in the analyses. This was verified by within-subjects ANOVAs that compared RTs for repeated and novel trials for NP and PC conditions as a function of SOA and spatial divergence of the target. All analyses failed to return any significant main effects or interactions involving repeated trials (all $F \mathrm{~s}<2.6$ ). Second, if participants were switching their attention from one cue to another between trials, the prediction is that RT variance for the cued trials in the spatially divergent case will be larger than the RT variance for the cued trials in the spatially convergent case. To test this we conducted within-subjects two-way ANOVAs on the NP and PC conditions separately, where each analysis contrasted the average variance for the cued trials in the spatially divergent case with the average variance for the cued trials in the spatially convergent case as a function of SOA. Both analyses returned no significant effects, demonstrating that the variances for the cued trials across SOAs are equivalent for spatially convergent and spatially divergent cases (all $F \mathrm{~s}<2$ ). Thus, the data converge on the conclusion that the effects elicited by spatially nonpredictive central cues reflect attentional orienting that occurs concurrent with, and independent from, orienting elicited by classic spatially nonpredictive peripheral onsets and spatially predictive symbolic cues.

\section{DISCUSSION}

This study sought to address and resolve the discrepancy between the experimental results and the theoretical accounts of attentional orienting elicited by central spatially nonpredictive cues. We measured the effects of central nonpredictive arrows and contrasted them with (1) the effects of nonpredictive peripheral onsets that engage exogenous orienting and (2) predictive central symbolic cues that engage endogenous orienting. If the effects of nonpredictive arrows were best conceptualized as exogenous, an interaction between the effects of nonpredictive arrow cues and nonpredictive peripheral cues would occur. If, on the other hand, the effects of nonpredictive arrows were best conceptualized as endogenous, an interaction with predictive central cues would occur. Finally, if the effects of nonpredictive arrow cues were best conceptualized as a hybrid between exogenous and endogenous orienting, interactions with both nonpredictive peripheral cues and central symbolic cues would occur.

Our results did not support any of these three predictions. We observed that the effects of a nonpredictive central arrow cue co-occurred with, and 
were independent of, both the endogenous orienting elicited by a predictive central cue and the exogenous orienting triggered by a nonpredictive peripheral onset. Four distinct lines of evidence support this conclusion. First, the attention effect observed for an arrow cue was the same whether the arrow was paired with an endogenous central cue or an exogenous peripheral cue. Second, the time course of the attention effect observed with the arrow cue was the same whether the arrow was paired with an endogenous central cue or an exogenous peripheral cue. Third, the attention effects when the cues indicated different spatial locations closely approximated the sum of the attention effects when the cues indicated the same spatial location. This convergence was observed across all SOAs for both endogenous and exogenous pairings. Finally, all of these effects emerged while presenting the two cues simultaneously rather than sequentially.

Taken together, our results carry important implications for both the prevailing theoretical accounts of attentional processes and future conceptualizations of human attention. Next we discuss first whether this new form of orienting, which is neither exogenous nor endogenous, is indicative of a new form of attentional control that draws on its own attentional "resources". We argue that it represents a unique form of attentional control; the issue of resources however remains uncertain. We then propose that this new form of orienting should be called automated symbolic orienting because it reflects an involuntary attentional response that has become automated as a function of repeated exposure to environmental contingencies.

Our findings offer firm evidence that orienting elicited by central nonpredictive cues operate independently from traditional exogenous and endogenous orienting. They also revive a long-standing issue regarding attentional control and the allocation of attentional resources (e.g., Berger et al., 2005; Klein, 2009; Muller \& Rabbitt, 1989). Although most researchers agree that exogenous and endogenous orienting differ in terms of the mechanisms of attentional control, there is as of yet no consensus about whether the two control mechanisms engage the same or separate resources (e.g., Berger et al., 2005; Klein, 2004, 2009; Luck, Hillyard, Mangun, \& Gazzaniga, 1994; Muller \& Rabbitt, 1989; Natale, Marzi, \& Macaluso, 2009). On balance, the prevailing view is that exogenous and endogenous orienting depend on separate attentional resources (see Klein, 2009, for a review that makes a compelling case for this position). This position is supported in part by behavioural data showing that exogenous and endogenous orienting exert qualitatively different effects on (1) perceptual processing (e.g., Briand \& Klein, 1987; Handy, Green, Klein, \& Mangun, 2001; Klein \& Hansen, 1987), (2) the formation of spatial and nonspatial expectancies (e.g., Klein \& Shore, 2000), (3) response compatibility (e.g., Lupianez et al., 2004), and (4) multisensory processing (e.g., Alais, Morrone, \& Burr, 2006). Similar conclusions follow from neuropsychological and neuroimaging studies (e.g., Corbetta \& 
Shulman, 2002; Hahn, Ross, \& Stein, 2006; Luck et al., 1994; Natale et al., 2009; Olk et al., 2010).

The opposing view maintains that while exogenous and endogenous attention are different in terms of the mechanisms of control they draw on the same underlying resource pool (Berger et al., 2005; Muller \& Humphreys, 1991; Muller \& Rabbitt, 1989). For example, in their classic study, Muller and Rabbit (1989) measured response accuracy and reported that while exogenous and endogenous orienting could co-occur, exogenous orienting could interrupt ongoing endogenous orienting, suggesting resource competition in which exogenous orienting had priority. Subsequently, Berger et al. (2005) qualified this conclusion, noting that exogenous and endogenous orienting usually operated independently, although mutual competition could briefly emerge when perceptual discrimination was difficult. Specifically, an invalid exogenous or endogenous cue modulated the attention effect of the competing cue but only at a $300 \mathrm{~ms}$ cue-target SOA, i.e., there was no interaction at the 100,200 , or $1000 \mathrm{~ms}$ cue-target SOAs.

Within this context, our data provide unequivocal support for the conclusion that orienting elicited by directional and spatially nonpredictive cues represents at least a unique type of attentional control. However, the question of whether this novel type of control draws on the same, or different, resources as the two classic modes remains to be answered. It has been suggested to us that a strong test of this hypothesis would involve the manipulation of a target's perceptual difficulty, similar to Berger et al.'s Experiment 4 (2005). However, this experimental manipulation does not provide a dissociation between the two theoretical positions for two key reasons. First, the interaction that Berger et al. reported was weak and transient, occurring at a single SOA of $300 \mathrm{~ms}$ and only when participants made errors on more than $15 \%$ of trials. Second, an interaction that arises with increasing task difficulty can also be attributed to other factors, such as changes in strategic set (as Berger et al. have themselves acknowledged) as well as to changes in the time that information is accessed (see van Zoest, Hunt, \& Kingstone, 2010).

Controlling for these concerns, we assessed attentional orienting using a speeded detection task, which allowed us to isolate attention without contaminating the data with response compatibility effects (Ristic, Wright, \& Kingstone, 2007) or oculomotor processes (Kuhn \& Kingstone, 2009; Posner, 1980). However, one might wonder whether the RT effects of a nonpredictive central arrow cue reflect changes in response bias rather than attentional orienting. There are at least three reasons to conclude that the effects that we have reported are attentional rather than due to response bias. First, a response bias interpretation does not predict our results. If response bias was a factor, one could expect that the attention effects created by either classic cue would interact with the response bias effect of the central 
nonpredictive arrow cue. For example, one would observe especially fast responses when a target appeared at a location that was validly cued by both stimuli, as that location would be both attended and have a lowered response criterion. In our study, the attention effects when the cues indicated different spatial locations closely approximated the sum of the attention effects when the cues indicated the same spatial location. Moreover this additivity was recorded for both endogenous and exogenous pairings with a central spatially nonpredictive arrow cue, and it was observed across each and every SOA interval. Second, whether combined with an exogenous or an endogenous cue, the cueing effects that we observed for nonpredictive arrows replicate behavioural results reported by past studies that have used either detection or discrimination tasks (e.g., Bayliss \& Tipper, 2006; Friesen \& Kingstone, 1998; Friesen, Ristic, \& Kingstone, 2004; Ristic et al., 2002; Tipples, 2002). Neuroimaging investigations carried out with EEG and fMRI further support the attentional explanation by showing that central cues measure attentional orienting and not response bias (e.g., Eimer, 1997; Tipper et al., 2008). This is evident in the typical attention-related modulation of target processing in extrastriate visual areas (i.e., the P1 modulation; e.g., Tipper et al., 2008) as well as in increased metabolic activity in the attentional control networks when central nonpredictive arrows are used (Hietanen, Nummenmaa, Nyman, Parkkola, \& Hamalainen, 2006; Tipper et al., 2008). Finally, Ivanoff and Saoud (2009, Experiment 3) explicitly tested whether the cueing effect of nonpredictive arrows produces a response bias. They failed to find any reliable evidence that central nonpredictive arrows lowered participants' response criteria, in that overall false alarm rate for arrow cues did not significantly differ from the false alarm rate produced by the classic peripheral cues. Thus, there is no evidence to support the notion that the effects of central nonpredictive arrow cues in our study reflect, even in a small part, the effects of response bias.

Ivanoff and Saoud (2009), however, reported that in contrast to arrows and classic peripheral cues, eye gaze cues, and pointing gestures can have a small and marginal effect on response bias, with participants committing on average $2.3 \%$ more false alarms on cued relative to uncued trials in those conditions. This result indicates that in general response bias does not explain attentional effects of central cues, and agrees with previous observations that differences can exist between the attentional effects elicited by different types of nonsocial behaviourally relevant (e.g., arrows, digits; Dodd, van der Stigchel, Leghari, Fung, \& Kingstone, 2008; Kingstone, Smilek, Ristic, Friesen, \& Eastwood, 2003) and social biologically relevant cues (e.g., eye gaze, finger point, head direction; Friesen et al., 2004; Nummenmaa \& Hietanen, 2009; Tipples, 2008). Attentional effects produced by biologically relevant stimuli, like eye gaze for instance, have been found to produce strongly automatic effects (i.e., attentional effects that are resistant to 
interruption and cue competition) relative to the effects of nonsocial behaviourally relevant cues, like arrows and central digits (e.g., Friesen et al., 2004; Ristic et al., 2006; Tipples, 2008). This dissociation between attentional effects of behaviourally and biologically relevant cues dovetails with other domains of investigation. For instance, neuroimaging studies report increased sensory processing (e.g., Tipper et al., 2008) as well as distinct neural activity in the attention networks associated with attending to eyes relative to attending to arrows (Hietanen et al., 2006), and neuropsychological investigations have found that eye gaze and arrows appear to involve different lateralized brain networks (Kingstone, Friesen, \& Gazzaniga, 2000; Ristic et al., 2002). Furthermore, studies of social attention conducted using more complex testing situations report that unlike common symbols, faces and facial features are more frequently selected and attended (e.g., Birmingham, Bischof, \& Kingstone, 2009), potentially revealing one of the key distinguishing features of biologically relevant social cues.

Thus, within the current framework of attentional processes, orienting elicited by central nonpredictive cues appears to be neither exogenous nor endogenous, where resistance to extraneous processes (i.e., the automaticity of the effect) may vary as a function of the type of the central cue. This distinction marks the crux of the current discrepancy between recent data and traditional attentional theory. To bridge this gap, we propose a terminology that reflects uniqueness of the behavioural performance profile observed with central cues (i.e., their ability to appear both exogenous and endogenous) and the distinctiveness of the types of cues that are found to elicit this effect (e.g., arrow direction, number magnitude, words with spatial meaning). Based on three well-documented findings, we suggest that these cues are best conceptualized as engaging automated symbolic orienting. First, the attentional effects elicited by central nonpredictive cues emerge rapidly, by $100 \mathrm{~ms}$, both when the cue is spatially uninformative (Frischen et al., 2007) and/or counterinformative of the target's position (Friesen et al., 2004; Tipples, 2008), thus displaying key properties of automatic processes (Hasher \& Zacks, 1979). Second, in contrast to automatic or exogenous orienting that is elicited by simple visual features (e.g., exogenous orienting elicited by luminance change), orienting in response to central cues is elicited by a cue that is symbolic and visually complex. Third, because the cue is symbolic, its meaning must be learned in order for it to become automatic. This notion is consistent with the idea of automatic processes that have developed through practice (e.g., Hasher \& Zacks, 1979). Along these lines, it has been proposed (e.g., Driver et al., 1999) that orienting elicited by central cues occurs as a result of long-term learning of the relevant contingencies that exist between the cue direction and target position in the environment, e.g., arrow direction is highly informative in terms of navigating our spatial environment. Once the learning of the associations between the cue and a 
probable event is established, the subsequent orienting proceeds automatically. Hence, the term "automated", which captures the learning aspect of this automatic effect. Thus, unlike endogenous orienting which occurs with intention and arises as a function of deliberate resource allocation, and exogenous orienting, which occurs without intention and arises as a function of simple sensory stimulation, automated symbolic orienting occurs without intention but arises as a function of the overlearning of the cue's contingency over time (e.g., Dodd \& Wilson, 2009; Hasher \& Zacks, 1979).

The point that orienting to behaviourally and biologically relevant cues is unique dovetails with a growing appreciation in cognitive neuroscience that the brain regions that are implicated in the control of attentional selection may be specialized to respond to nonsocial behaviourally relevant information (Kincade, Abrams, Astafiev, Shulman, \& Corbetta, 2005; Ristic \& Giesbrecht, 2011) and social biologically relevant information (Corbetta, Patel, \& Shulman, 2008). Whereas past studies have argued that the brain mechanisms implicated in the control of exogenous and endogenous orienting dissociate both anatomically and functionally (e.g., Corbetta \& Shulman, 2002), more recent research points to an increased complexity in the neural networks associated with attentional control and their involvement in social cognition (Corbetta et al., 2008). Traditionally, exogenous orienting has been associated with subcortical reflex circuits that include the superior colliculus and the pulvinar (e.g., Rafal, Posner, Friedman, Inhoff, \& Bernstein., 1988; Wilke, Turchi, Smith, Mishkin, \& Leopold, 2010) as well as the regions of the right-lateralized ventrolateral frontoparietal attention network (Corbetta, Kincade, Ollinger, McAvoy, \& Shulman, 2000), whereas endogenous orienting has been associated with cortical circuits that form the dorsolateral frontoparietal attention network (Corbetta \& Shulman, 2002). More recent studies, however, point to an increased role of the dorsolateral network in attentional selection regardless of whether attention has been committed exogenously or endogenously (Corbetta et al., 2008), and the structures of the ventrolateral network are increasingly being implicated as playing a large role in mediating attentional orienting to behaviourally relevant events and biologically relevant social stimuli (Corbetta et al., 2008; Kincade et al., 2005; Ristic \& Giesbrecht, 2011). Arrows fall firmly within the domain of behaviourally relevant cues, in that while they are spatially nonpredictive in experiments such as the present one, in real-life situations they convey highly meaningful information. Neuroimaging studies conducted to date that have investigated neural mechanisms associated with orienting elicited by nonsocial behaviourally relevant cues suggest unique activity both in terms of spatial and temporal activity in the cortical regions comprising the ventrolateral attention network (e.g., Hietanen et al., 2006; Ristic \& Giesbrecht, 2011). Specifically, attentional orienting to behaviourally relevant cues appears to uniquely engage the areas of the frontal lobe 
(i.e., middle frontal gyrus-MFG; Ristic \& Giesbrecht, 2011) and occipitotemporal cortex (i.e., temporoparietal junction-TPJ; Kincade et al., 2005), which are critical not only for switching between attentional networks but also for tracking and responding to behaviourally relevant contingencies in the environment (e.g., Corbetta et al., 2008). Of course, since both behaviourally relevant and biologically relevant stimuli are highly relevant for complex behaviours, several neuroimaging studies have also observed overlapping neural activation in the areas traditionally associated with attentional control (e.g., Greene et al., 2009; Tipper et al., 2008). Future studies are needed to precisely elucidate the neural mechanisms that are implicated in the attentional control of behaviourally relevant and biologically relevant cues.

In sum, using a novel simultaneous dual-cue attention paradigm our study revealed that automated symbolic orienting is a unique attentional control mechanism that is different from classic forms of exogenous and endogenous orienting in terms of its behavioural outcomes and potentially, as suggested by past studies, its underlying neural mechanisms. Our results also suggest that the classic distinction between exogenous and endogenous attentional control is not comprehensive enough to capture the richness of attentional processes that can occur when participants encounter highly meaningful stimulus inputs. This idea is consistent with a recent proposal that the neural networks implicated in the control of attention are fundamentally influenced by the behavioural relevance of available sensory information (Corbetta et al., 2008; Ristic \& Giesbrecht, 2011). We believe that conceptualizing attentional processes within this expanded framework will provide a fruitful ground for future investigations of attention.

\section{REFERENCES}

Alais, D., Morrone, C., \& Burr, D. (2006). Separate attentional resources for vision and audition. Proceedings of the Royal Society B: Biological Sciences, 273(1592), 1339-1345.

Bartolomeo, P., \& Chokron, S. (2002). Orienting of attention in left unilateral neglect. Neuroscience and Biobehavioral Reviews, 26(2), 217-234.

Bayliss, A. P., \& Tipper, S. P. (2006). Predictive gaze cues and personality judgments: Should eye trust you? Psychological Science, 17(6), 514-520.

Berger, A., Henik, A., \& Rafal, R. (2005). Competition between endogenous and exogenous orienting of visual attention. Journal of Experimental Psychology: General, 134(2), 207-221.

Bertelson, P. (1967). The time course of preparation. Quarterly Journal of Experimental Psychology, 19(3), 272-279.

Birmingham, E., Bischof, W., \& Kingstone, A. (2009). Get real! Resolving the debate about equivalent social stimuli. Visual Cognition, 17(6), 904-924.

Bonato, M., Priftis, K., Marenzi, R., \& Zorzi, M. (2008). Modulation of hemispatial neglect by directional and numerical cues in the line bisection task. Neuropsychologia, 46(2), $426-433$. 
Briand, K. A., \& Klein, R. M. (1987). Is Posner beam the same as Treisman glue - on the relation between visual orienting and feature integration-theory. Journal of Experimental Psychology: Human Perception and Performance, 13(2), 228-241.

Brodeur, D., Trick, L. M., \& Enns, J. T. (1997). Selective attention over the lifespan. In J. A. Burack \& J. T. Enns (Eds.), Attention, development, and psychopthology (pp. 74-94). New York, NY: Guilford Press.

Corbetta, M., Kincade, J. M., Ollinger, J. M., McAvoy, M. P., \& Shulman, G. L. (2000). Voluntary orienting is dissociated from target detection in human posterior parietal cortex. Nature Neuroscience, 3(3), 292-297.

Corbetta, M., Patel, G., \& Shulman, G. L. (2008). The reorienting system of the human brain: From environment to theory of mind. Neuron, 58(3), 306-324.

Corbetta, M., \& Shulman, G. L. (2002). Control of goal-directed and stimulus-driven attention in the brain. Nature Reviews Neuroscience, 3(3), 201-215.

Deaner, R. O., \& Platt, M. L. (2003). Reflexive social attention in monkeys and humans. Current Biology, 13(18), 1609-1613.

Dodd, M. D., van der Stigchel, S., Leghari, M. A., Fung, G., \& Kingstone, A. (2008). Attentional SNARC: There's something special about numbers (let us count the ways). Cognition, 108(3), 810-818.

Dodd, M. D., \& Wilson, D. (2009). Training attention: Interactions between central cues and reflexive attention. Visual Cognition, 17(5), 736-754.

Dorris, M. C., Klein, R. M., Everling, S., \& Munoz, D. P. (2002). Contribution of the primate superior colliculus to inhibition of return. Journal of Cognitive Neuroscience, 14(8), $1256-1263$.

Driver, J., Davis, G., Ricciardelli, P., Kidd, P., Maxwell, E., \& Baron-Cohen, S. (1999). Gaze perception triggers reflexive visuospatial orienting. Visual Cognition, 6(5), 509-540.

Egeth, H. E., \& Yantis, S. (1997). Visual attention: Control, representation, and time course. Annual Review of Psychology, 48, 269-297.

Eimer, M. (1997). Uninformative symbolic cues may bias visual-spatial attention: Behavioral and electrophysiological evidence. Biological Psychology, 46(1), 67-71.

Fischer, M. H., Castel, A. D., Dodd, M. D., \& Pratt, J. (2003). Perceiving numbers causes spatial shifts of attention. Nature Neuroscience, 6(6), 555-556.

Friesen, C. K., \& Kingstone, A. (1998). The eyes have it! Reflexive orienting is triggered by nonpredictive gaze. Psychonomic Bulletin and Review, 5(3), 490-495.

Friesen, C. K., Ristic, J., \& Kingstone, A. (2004). Attentional effects of counterpredictive gaze and arrow cues. JEP: Human Perception and Performance, 30(2), 319-329.

Frischen, A., Bayliss, A. P., \& Tipper, S. P. (2007). Gaze cueing of attention: Visual attention, social cognition, and individual differences. Psychological Bulletin, 133(4), 694-724.

Gibson, B. S., \& Kingstone, A. (2006). Visual attention and the semantics of space: beyond central and peripheral cues. Psychological Science, 17(7), 622-627.

Graham, R., Friesen, C. K., Fichtenholtz, H. M., \& LaBar, K. S. (2010). Modulation of reflexive orienting to gaze direction by facial expressions. Visual Cognition, 18(3), $331-368$.

Green, J. J., \& Woldorff, M. G. (2012). Arrow-elicited cueing effects at short intervals: Rapid attentional orienting or cue-target stimulus conflict? Cognition, 122(1), 96-101.

Greene, D. J., Mooshagian, E., Kaplan, J. T., Zaidel, E., \& Iacoboni, M. (2009). The neural correlates of social attention: automatic orienting to social and nonsocial cues. Psychological Research, 73(4), 499-511.

Hahn, B., Ross, T. J., \& Stein, E. A. (2006). Neuroanatomical dissociation between bottom-up and top-down processes of visuospatial selective attention. Neuroimage, 32(2), 842-853.

Handy, T. C., Green, V., Klein, R. M., \& Mangun, G. R. (2001). Combined expectancies: Eventrelated potentials reveal the early benefits of spatial attention that are obscured by reaction 
time measures. Journal of Experimental Psychology: Human Perception and Performance, 27(2), 303-317.

Hasher, L., \& Zacks, R. T. (1979). Automatic and effortful processes in memory. Journal of Experimental Psychology: General, 108(3), 356-388.

Hietanen, J. K., Nummenmaa, L., Nyman, M. J., Parkkola, R., \& Hamalainen, H. (2006). Automatic attention orienting by social and symbolic cues activates different neural networks: An fMRI study. NeuroImage, 33(1), 406-413.

Hood, B. M., Willen, J. D., \& Driver, J. (1998). Adult's eyes trigger shifts of visual attention in human infants. Psychological Science, 9, 53-56.

Ivanoff, J., \& Saoud, W. (2009). Nonattentional effects of nonpredictive central cues. Attention, Perception and Psychophysics, 71(4), 872-880.

James, W. (1890). The principles of psychology. New York, NY: Dover Publications.

Jonides, J. (1981). Voluntary versus automatic control over the mind's eye's movement. In J. B. Long \& A. D. Baddeley (Eds.), Attention and performance IX (pp. 187-203). Hillsdale, NJ: Lawrence Erlbaum Associates.

Kincade, J. M., Abrams, R. A., Astafiev, S. V., Shulman, G. L., \& Corbetta, M. (2005). An event-related functional magnetic resonance imaging study of voluntary and stimulus-driven orienting of attention. Journal of Neuroscience, 25(18), 4593-4604.

Kingstone, A., Friesen, C. K., \& Gazzaniga, M. S. (2000). Reflexive joint attention depends on lateralized cortical connections. Psychological Science, 11(2), 159-166.

Kingstone, A., Smilek, D., Ristic, J., Friesen, C. K., \& Eastwood, J. D. (2003). Attention, researchers! It is time to take a look at the real world. Current Directions in Psychological Science, 12, 176-180.

Klein, R. (2009). Canadian Laboratories/Laboratoires Canadiens on the control of attention. Canadian Journal of Experimental Psychology-Revue Canadienne De Psychologie Experimentale, 63(3), 240-252.

Klein, R., \& Hansen, E. (1987). Spotlight failure in covert visual orienting. Bulletin of the Psychonomic Society, 25(6), 447-450.

Klein, R., \& Shore, D. I. (2000). Relations among modes of visual orienting. In S. Monsell \& J. Driver (Eds.), Control of cognitive processes (pp. 195-208). Cambridge, MA: MIT Press.

Klein, R. M. (2004). On the control of visual orienting. In M. I. Posner (Ed.), Cognitive neuroscience of attention (pp. 29-45). New York, NY: Guilford Press.

Kuhn, G., \& Kingstone, A. (2009). Look away! Eyes and arrows engage oculomotor responses automatically. Attention, Perception and Psychophysics, 71(2), 314-327.

Langton, S. R. H., \& Bruce, V. (1999). Reflexive visual orienting in response to the social attention of others. Visual Cognition, 6(5), 541-567.

Luck, S. J., Hillyard, S. A., Mangun, G. R., \& Gazzaniga, M. S. (1994). Independent attentional scanning in the separated hemispheres of split-brain patients. Journal of Cognitive Neuroscience, 6(1), 84-91.

Lupianez, J., Decaix, C., Sieroff, E., Chokron, S., Milliken, B., \& Bartolomeo, P. (2004). Independent effects of endogenous and exogenous spatial cueing: Inhibition of return at endogenously attended target locations. Experimental Brain Research, 159(4), 447-457.

McKee, D., Christie, J., \& Klein, R. (2007). On the uniqueness of attentional capture by uninformative gaze cues: Facilitation interacts with the Simon effect and is rarely followed by IOR. Canadian Journal of Experimental Psychology, 61(4), 293-303.

Muller, H. J., \& Humphreys, G. W. (1991). Luminance-increment detection: Capacity-limited or not? Journal of Experimental Psychology: Human Perception and Performance, 17(1), 107-124.

Muller, H. J., \& Rabbitt, P. M. (1989). Reflexive and voluntary orienting of visual attention: Time course of activation and resistance to interruption. Journal of Experimental Psychology: Human Perception and Performance, 15(2), 315-330. 
Natale, E., Marzi, C. A., \& Macaluso, E. (2009). FMRI correlates of visuo-spatial reorienting investigated with an attention shifting double-cue paradigm. Human Brain Mapping, 30(8), $2367-2381$.

Nummenmaa, L., \& Hietanen, J. K. (2009). How attentional systems process conflicting cues: The superiority of social over symbolic orienting revisited. Journal of Experimental Psychology: Human Perception and Performance, 35(6), 1738-1754.

Olk, B., Hildebrandt, H., \& Kingstone, A. (2010). Involuntary but not voluntary orienting contributes to a disengage deficit in visual neglect. Cortex, 46(9), 1149-1164.

Posner, M. (1980). Orienting of attention. Quarterly Journal of Experimental Psychology, 32(1), $3-25$.

Posner, M. I. (1978). Chronometric explorations of mind. Hillsdale, NJ: Lawrence Erlbaum Associates.

Posner, M. I., \& Cohen, Y. (1984). Components of visual orienting. In H. Bouma \& D. G. Bouwhuis (Eds.), Attention and performance X: Control of language processes (pp. 531-566). Hillsdale, NJ: Lawrence Erlbaum Associates.

Rafal, R. D., Posner, M. I., Friedman, J. H., Inhoff, A. W., \& Bernstein, E. (1988). Orienting of visual attention in progressive supranuclear palsy. Brain, 111, 267-280.

Ristic, J., Friesen, C. K., \& Kingstone, A. (2002). Are eyes special? It depends on how you look at it. Psychonomic Bulletin and Review, 9(3), 507-513.

Ristic, J., \& Giesbrecht, B. (2011). Electrophysiological evidence for spatiotemporal flexibility in the ventrolateral attention network. PLoS ONE, 6(9), e24436.

Ristic, J., \& Kingstone, A. (2006). Attention to arrows: Pointing to a new direction. Quarterly Journal of Experimental Psychology, 59(11), 1921-1930.

Ristic, J., Mottron, L., Friesen, C. K., Iarocci, G., Burack, J. A., \& Kingstone, A. (2005). Eyes are special but not for everyone: The case of autism. Cognitive Brain Research, 24(3), $715-718$.

Ristic, J., Wright, A., \& Kingstone, A. (2006). The number line effect reflects top-down control. Psychonomic Bulletin and Review, 13(5), 862-868.

Ristic, J., Wright, A., \& Kingstone, A. (2007). Attentional control and reflexive orienting to gaze and arrow cues. Psychonomic Bulletin and Review, 13, 862-868.

Schuller, A. M., \& Rossion, B. (2001). Spatial attention triggered by eye gaze increases and speeds up early visual activity. Neuroreport, 12(11), 2381-2386.

Taylor, T. L., \& Klein, R. M. (1998). On the causes and effects of inhibition of return. Psychonomic Bulletin and Review, 5(4), 625-643.

Tipper, C. M., Handy, T. C., Giesbrecht, B., \& Kingstone, A. (2008). Brain responses to biological relevance. Journal of Cognitive Neuroscience, 20(5), 879-891.

Tipples, J. (2002). Eye gaze is not unique: Automatic orienting in response to uninformative arrows. Psychonomic Bulletin and Review, 9(2), 314-318.

Tipples, J. (2008). Orienting to counterpredictive gaze and arrow cues. Perception and Psychophysics, 70(1), 77-87.

Van Zoest, W., Hunt, A. R., \& Kingstone, A. (2010). Representations in visual cognition: It's about time. Current Directions in Psychological Science, 19(2), 116-120.

Vecera, S. P., \& Rizzo, M. (2006). Eye gaze does not produce reflexive shifts of attention: Evidence from frontal-lobe damage. Neuropsychologia, 44(1), 150-159.

Wilke, M., Turchi, J., Smith, K., Mishkin, M., \& Leopold, D. A. (2010). Pulvinar inactivation disrupts selection of movement plans. Journal of Neuroscience, 30(25), 8650-8659. 